\title{
ART Chewing Gum: A Linkage to Care
}

\author{
Rahul Hajare* \\ ICMR Post Doc Indian Council of Medical Research New Delhi, India
}

Received: May 26, 2018; Published: May 31, 2018

*Corresponding author: Rahul Hajare, ICMR Post Doc Indian Council of Medical Research New Delhi, India

\begin{abstract}
The oral effects of HIV and AIDS occur because of your weakened immune system and the medicines that kept under control. These effects have also found in other people with weakened immune systems. Dry mouth (xerostomia) because people with HIV have an increased risk of cavities, dentist will provide a program to reduce the risk. This may include fluoride varnishes applied in the dental office, toothpastes that add minerals to teeth and rinses that contain fluoride or reduce acid (acidity of mouth) in the mouth. Patient education has an essential part of this program. Dry mouth has xerostomia has a symptom of HIV infection but not something that would be used as a diagnostic tool. There are many reasons if you have HIV, changes in mouth may reflect changes in immune status. Dry mouth (xerostomia) has a common side effect of medicines that may take for HIV/AIDS. Dry mouth can make more prone to tooth decay, gingivitis, thrush (oral yeast infection) and periodontal (gum) disease. That's because have less saliva, and the quality of the saliva is changed exponentially. Besides water, saliva includes electrolytes, mucus, antibacterial compounds, enzymes and other proteins. The amounts of these substances change in people with dry mouth. A decrease in saliva reduces its ability to wash away sugars, food, bacteria and the acids that bacteria produce. These acids cause teeth to decay. Dentist can prescribe a fluoride rinse or gel or a saliva substitute to counteract the increased risk of tooth decay and thrush.
\end{abstract}

\section{Introduction}

HIV patients cannot produce saliva properly which is required to convert immune system, and other food source into energy. Researcher has been looking for simple and effective ways to deliver ART into the blood stream. The most common one has taking ART pill orally. However research shows that ART has easily broken down by adverse drug reaction of dry mouth and tong. This problem has solved by using chewing gum ART formulation. These can be done by binding ART with saliva. The saliva is protected with salivary protein. Once saliva ART reaches the intestines, another chemical pathway takes over to help ART pass into the bloodstream. Binding of ART molecules to saliva makes the ART hitch a ride on this protected supply chain. The ART could ride all the way into the bloodstream, where it is released to do its work. Chewing gum also causes stimulation of brain which leads to increased release of anti-depression drug. Finding simpler ways to deliver ART into the blood stream is one important avenue for tackling the dry mouth rashes of HIV patient epidemic that is sweeping the developed world. Body has specific mechanisms for protecting and absorbing valuable molecules that would usually be damaged by conditions in the gut. Chewing gum would be a better delivery method in humans living with HIVAIDS [1-5].

\section{How Chewing Gum Works}

Man has a habit of chewing the chewing gum since ancienttimes. Today it has one of the most popular dosage form, used for delivering the many active components. The first medical chewing gum was introduced in market in 1928 consisting of aspirin an analgesic drug. However, chewing gum did not gain acceptance as a reliable drug delivery system until 1978, when nicotine chewing gum became available in 1980. Most of the chewing gum has used for smoking cessation (containing the nicotine) and also used for oral and dental hygiene (consisting of fluoride and carbamide etc). Today, medical chewing gum meets the same high-quality standards as tablets and can be formulated to obtain different release profiles of active substances, thus enabling distinct patient group targeting. It can be taken discreetly without water and allows for local and systemic delivery. It can be employed for treatment of diseases of the oral cavity and throat, e.g. for caries prevention, or it can release drugs that can be absorbed through oral mucosa directly into the systemic circulation. In addition, drug that is not absorbed by the oral cavity membranes can be dissolved in the saliva before swallowing, thus leading to a more rapid onset of action, less side effect [6-9].

The majority of chewing gum delivery systems today have manufactured using conventional gum processes. The gum base is softened or melted and placed in a kettle mixer where sweeteners, syrups, active ingredients and other excipients are added at a defined time. The gum can then sent to a series of rollers that form it into a thin, wide ribbon. During this process, a light coating of finely 
powdered sugar or sugar substitute is added to keep the gum from sticking and to enhance the flavors. Finally, the gum is cut to the desired size and cooled at a carefully controlled temperature and humidity. The need for and value of in vitro drug release testing has well established for a range of dosage forms, however, standard dissolution apparatus has not suitable for monitoring release of drug from chewing gums as the action of chewing is essential, by providing a renewable surface for drug release after chewing action. The release of substances from chewing gums during mastication can be studied by employing a panel of tasters and chew-out studies. During the mastication process, the medication contained within the gum product should be released into the saliva and either absorbed through the oral mucosa or swallowed and absorbed through the gastrointestinal tract. The chewed gum can then be removed and analyzed for the residual drug substance while pharmacokinetics can be determined from blood a sample that helps to examination of human immunity recovery [9-11].

\section{Why we use ART Chewing Gum as A Drug Delivery Sys- tem?}

a) Easy for administration without water promotes higher patient compliance.

b) Children and for patients who find swallowing tablets difficult are obvious.

c) Fast onset of action

d) Less side effects

e) Effective on dry mouth.

f) Effective on dry tongue.

g) Local therapy.

h) Cost effective

i) Less chemical data required for clinical trial.

\section{Mechanism of Action}

Past studies have shown that the digestive system have associated with gastrointestinal disturbances, headaches, hyperglycaemia (caution in diabetes), increased risk of bleeding of ART, and that any surviving enzyme is not easily absorbed into the bloodstream from the gut. Mammals have a transport mechanism for the absorption and cellular uptake of the relatively large essential saliva molecule which relies upon complexing to a naturally occurring transport protein known as intrinsic factor such as salivary protein. Chewing anything produces saliva, which triggers the release of immunity system. Combination of chewing gum with ART and protein are given during mastication process which leads to production of saliva which containing hepatocorrin protein which coat the vitamin B series and act as intrinsic factor for absorption of vitamin B coating ART into blood stream vitamin B protect the ART from digestive enzyme. Active substances like chromium, guaran and caffeine are proved to be efficient in treating ART's. Chromium is claimed to reduce craving for food due to an improved blood-glucose balance while patient having low BMR profile. Caffeine and guaran stimulate lipolysis and have a thermogenic effect (increased energy expenditure) and increase feeling of hunger.

\section{Statement of Problem}

Researcher from globe of technology has developed a onceweekly pill package that stays in the stomach to deliver a gradual long-lasting dose of medications to combat HIV. Studies have shown that around $50 \%$ of patients do not take medicine for chronic diseases or conditions as prescribed, thereby reducing the effectiveness of treatment.

\section{Conclusion}

ART chewing gum is an excellent drug delivery system for easy-medication, also reduce the hesitation of people living with HIV/aids, as it is convenient and can be administered discreetly with-out water, without fear, without hesitation. It offers removal of needles fear from the patient. ART Chewing gum helps to give ART orally for HIV people living with diabetics and tackle the degradation of ART by digestive enzyme without adding digestive enzyme inhibitor. It helps to reduce the cost of therapy \& improve the brain boost power, concentration \& smoking cessation. The release of ART chewing gum leads to an increased heart rate and sends glucose and oxygen to our brain. The glucose and oxygen in our brain helps improve concentration, focus, and learning can reduce depression sign of people living HIV AIDS. An ART chewing gum can offer a significant solution to the breaking down of orally-taken ART by the digestive system can reduce irritation and dose frequency.

\section{Conflicts of Interest}

The author declares that there were no conflicts of interest to report, research funding. This study has purely new and projected as an empirical study for use in development of easy and reasonable drug delivery for people living with HIV AIDS. The purpose of this unfunded project for health management, improvement of HIV medication adherence. A medical care and protection of family circle. This article belongs to the Public Health. The experimental data and analysis part is very sufficient, and the conclusion is very reasonable and understandable. It is recommended to publish the article in your esteemed journal. I hope this helps.

\section{References}

1. Ramesh Paranjape, Rahul Hajare, Smita Kulkarni (2016) Tailored microwave technology for Synthesis N'-[(3Z)-5-chloro-1-(morpholin4-ylmethyl)-2-oxo-1,2-dihydro-3H-indol-3ylidene]pyridine-4 carbohydrazide as HIV-1 inhibitors. Drug Formulation \& Bioavailability Congress" 05.

2. Ramesh Paranjape, Rahul Hajare, Ramesh, Smita Kulkarni (2016) Technology development and design of novel 1,3,5-tri substituted-1Hindole-2,3-dione: HIV-1 Inhibitors with displays strategic nanomolar cytotoxicity. (2016 )251stACS National Meeting \& Exposition held on March 13-17, in San Diego, CA, USA

3. Ramesh P, Rahul H, Smita K, Madhuri T (2016) Variability interaction between Etravirine and Rilpivirine: a Retrospective. KJACT -100103 2: $1-3$.

4. Ramesh Paranjape, Rahul Hajare, Smita Kulkarni, Madhuri Thakar (2016) Technology Development and Design of Novel 1, 3, 5-tri Substituted-1HIndole-2, 3-Dione HIV-1 Inhibitors With Displays Strategic Nanomolar Cytotoxicity. World J Pharm Pharm Sci 5(6): 391. 
5. Ramesh Paranjape, Rahul Hajare, Smita Kulkarni, Madhuri (2016) Isatin Anti-HIV Agent: A Review. World J Pharm Pharm Sci 5: 569-575.

6. Ramesh Shivram Paranjape, Rahul Anandrao Hajare, Smita S (2015) Kulkarni, Design Space Filling Model, Synthesis and Evaluation of Novel 2-Indolinone HIV-1 Inhibitors. International Journal of Advanced Research 3(12): 1332-1335.

7. Rahul Hajare, New heights of antiretroviral drug delivery (2017) 22nd International Conference and Expo on Nanoscience and Molecular Nanotechnology 06-08, Germany.

8. Ramesh Paranjape, Rahul Hajare (2017) New Technology of Nucleoside AIDS Virus Resistance Drug Key Intermediate Diethyl (Tosyloxy) Methylphosphonate. $11^{\text {th }}$ World Congress on Pharmaceutical Sciences and Innovations in Pharma Industry . Amsterdam, Netherlands.
9. Ramesh Paranjape, Rahul Hajare (2017) Nanotechnology- in HIV/ AIDS Therapy. International Conference on Advanced Nanotechnology September 11-12, Amsterdam, Netherlands.

10. Ramesh Paranjape, Rahul Hajare (2017) New developments in adverse drug reaction of ART and tertiary care. 8th Global Pharmacovigilance \& Drug Safety Summit. Kuala Lumpur, Malaysia.

11. Rahul Hajare (2017) New pattern clinical study of adverse drug reaction to ART may lead to chase drug discovery in anti-retroviral drug towards zero adverse drug reaction. 4th International Conference on Clinical Trials 11-13, San Antonio, Texas, USA.
(C) (P) This work is licensed under Creative

Submission Link: https://biomedres.us/submit-manuscript.php

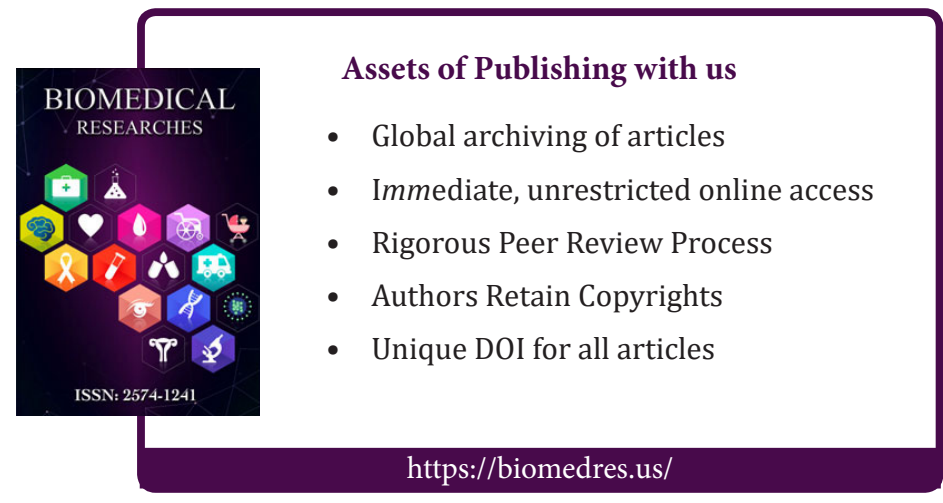

